\title{
BRAIN TUMOR DETECTION IN MAGNETIC RESONANCE IMAGES
}

Balkrishan Jindal $^{l} \&$ Damanpreet Singh ${ }^{2}$

Abstract- Magnetic Resonance Imaging (MRI) of the brain is a painless and secure method in medical field. It is used to create images of human body for diagnosis of various diseases in the human body. In this paper, brain tumor is detected based on Gray level co-occurrence matrix used for computation of energy, contrast and homogeneity. Segmentation is used to detect location, shape and size of the brain tumor in MRI images. These features are further used for training the Feed Forward Neural Network (FFNN) for classify the image. The performance of the proposed method evaluated using parameters like Area, Perimeter, Contrast, Energy, Homogeneity, Mean Square Error and Confusion Matrix. The experimental results of the proposed method are also compared with Back Propagation Neural Network. From the experimental results it has been concluded that the performance parameters of the proposed method are better than Back Propagation Neural Network.

Keywords: MRI Brain Tumour, Image Segmentation, Feature Extraction, Feed Forward Neural Network, Mean Square Error.

\section{INTRODUCTION}

Magnetic Resonance Images are commonly used for diagnosing brain tumor with the help of scan based image processing technique. The normal MRI images are not suitable for accurate analysis, so segmentation is used for effective analyzing of brain tumor images [1]. Image segmentation is vital and demanding factor in the area of medical science. Segmentation is used to detect location, shape and tumor size in magnetic resonance images. There are various methods and algorithms like Boundary based, Threshold based and Region based are used to detect brain tumor from MRI images [2,3].

Brain Tumor is mass of unwanted tissues which rapidly grow in brain with normal cells. Neural Network, Fuzzy algorithm and Knowledge based methods are used for analyzing inner part of brain. Brain tumor is divided into Primary or Metastatic and Begin or Maligant tumors. Primary or Metastatic tumors are developed in the brain itself. Begin or Maligant tumors are developed in the body and reached the brain [1].

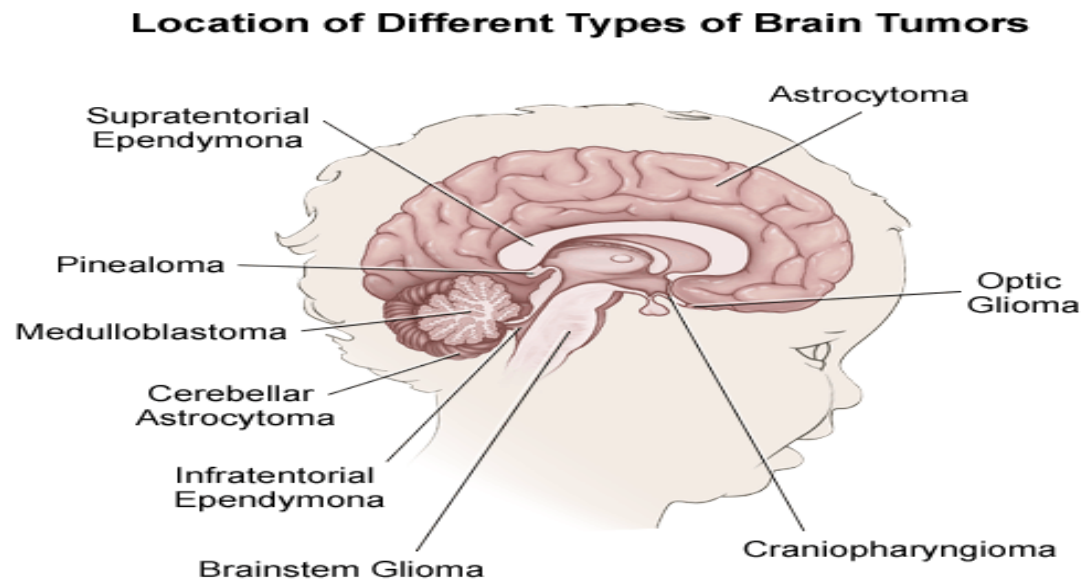

Figure 1: MRI Image of the Brain Tumor [4]

The symptoms of each type of brain tumor depend on the part of brain. The headache is classically severe in the morning and replace with vomiting, afterwards. More special problem includes difficulties with walking, speaking and with sensation. As the disease progresses, there can be an unconsciousness $[4,5]$.

${ }^{1}$ Assistant Professor, CE Section, Yadavindra College of Engineering, Guru Kashi Campus, Punjabi University, Talwandi Sabo, Bathinda, Punjab (India)

${ }^{2}$ Student, Yadavindra College of Engineering, Guru Kashi Campus, Punjabi University, Talwandi Sabo Bathinda, Punjab (India) 
In 2017, approximately 80,000 cases of brain tumors are detected. Near about 30\% of Central Nervous System (CNS) and brain tumor found to be Malignant which includes more than 26,000 Primary and 53,000 non malignant tumors. In this year, around 17,000 people will lose their lives due to malignant and CNS brain tumor. Existence after detection with primary tumor varies by behavior of tumor, histology, age and markers. There are near about 100 types of different primary and CNS tumor detected. In the recent research, it is predicted that 4800 case in children and adolescent will face primary tumor in this year $[6,7]$.

\section{IMAGE SEGMENTATION}

It is the method of partitioning a medical image into different parts/ segments. The main motive of the image segmentation is to convert the representation and clarify the extraction of images to analyze and more meaningful information for medical purpose. Segmentation is utilized to locate curves, lines and objects in medical images [8].

\subsection{Methods of Segmentation}

There are various methods used for segmentation which are written as follows [8]

\subsubsection{Thresholding}

It is the easiest strategy used in image segmentation. This method converts gray scale into binary value. It is based on threshold value or also called as clip-level value. In thresholding, morphological operations are essentially based on some assumptions regarding the dimensions and form of the brain tumor [1].

\subsubsection{Clustering methods}

It is the process of dividing the whole image into small parts also called k-clusters using $\mathrm{k}$-means algorithm. $\mathrm{K}$ is a positive integer number. Different steps used in the algorithm are as follows.

1. Pick centers of k-clusters, either it depend on heuristic or separately

2. Compute the cost between cluster centre and data point

3. Re-compute centers of the clusters.

4. Repeat second and third stage until converges.

\subsubsection{Histogram based method}

Histogram based strategies are useful when compared to other image segmentation strategies. It is used to convert cluster in the medical image into small clusters. The main disadvantage of this method is that it can be difficult to verify valleys and peaks in the medical image.

\subsubsection{Compression based method}

Compression based strategy is used for optimal segmentation that minimize coding length of the structure and over all hopeful segmentation. With the help of two ideas segmentation tries to seek out patterns and any regularity within the image are often used to compress it. Boundary shape and texture feature is evaluated with the help of compression method.

\subsubsection{Region growing method}

This method is used to compute the neighboring pixel of seed points and that are adding to the region of image. Firstly, it takes a collection of seed points as input along with the image and seed marked every objects is to be divided. With the help of region growing method region is directly computed.

\subsubsection{Edge Detection}

Edge detection is a fundamental strategy utilized for evaluating the boundaries of the image. Edges and region based boundaries are close to each other. This method must applicable to obtain edges from edge detector which employs clustering technique [8].

\section{LITERATURE REVIEW}

Somwanshi et al. [2] explained the entropy measurements that utilized to obtain efficient performance in brain tumor detection from magnetic resonance images. Segmentation is mainly used to detect shape, size and location of the brain tumor. Threshold value, Boundary based and Region based methods are employed for segmentation. Havrda charvat and Kapur, Shannon and Vajda entropy techniques are measure the entropy of the MRI image. Havrda charvat entropy calculates best performance than other entropy methods.

Parveen and Agarwal [4] explained the existing automatic, manual and semi automatic methods for medical imaging. This paper contains four steps used for detecting the brain tumor and to evaluate features from magnetic resonance images. First step comprises skull detection and noise filtering. Second step used for GLCM. Third step explains SVM for Perceptron kernel 
and fourth step using fast bounding box for segmentation of tumor part in brain. In the last it can be concluded that this method provide better results than previous techniques.

Kaur and Bhatia [8] introduced the Pollination and Penalized FCM approach used in segmentation for medical images. There are six segmentation methods namely Compression based, Histogram based, Clustering, Region growing, Edge detection and Thresholding. With the help of Threshold value gray scale picture is transfer into binary picture. The main aim of this technique is medical images which are used for segmentation.

Kaur [9] explained the various segmentation methods used for brain tumor extraction. Brain tumor is a life threatening and inherently serious disease that can destroy human life. In this paper Fuzzy c means, K means \& Thresholding methods used for brain tumor extraction. In this work data set of $25 \mathrm{MRI}$ images has been used for testing and obtaining the effective results. Fuzzy c means is combined with Morphological components for evaluating best result. FCM method gives $90.57 \%$ that is better than Morphological method.

Sehgal et al. [10] explained the segmentation of Magnetic Resonance Images for brain tumor detection based on Fuzzy C Means method. They also discussed the preprocessing methods like Anisotropic diffusion filtering method, Bias correction and Sharpening of image to detect brain tumor from MRI images. They evaluate performance of their method by measured the Dice coefficient and showed the average value of the Dice coefficient is 0.729 .

Bhima and Jagan [11] discussed the Watershed algorithm for gray scale MRI images based on region direction and mathematically morphologically. Segmentation method is used to identify the brain tumor in MRI images. Fuzzy c-means, CRF, SVM, Localization and Multi-region feature selection and merits of Watershed methods are analyzed with possible enhancement.

Chuan Long Li et al [12] explained the Novel Fuzzy C-means which is used for Spatial Neighborhoods in digital image. It mainly involves two process first is replacement of Euclidean distance utilize for measuring similarity and second is not required for any similarity. Till now no method fully satisfied all the medical image segmentation but Fuzzy c-means method is well known only used for clustering. This procedure does not evaluate any specific parameters.

Al-Azzawi and Sabir [13] explained the Fuzzy transform segmentation which is used in brain tumor detection to handle the information and salient edges. There are two steps used in this method. First step is symmetrical and asymmetry study related to brain and existence of tumor is measured by gray level in symmetrical. Second step is for edge detection. Morphological operations also used in this technique. The performance of precision is $95 \%$ and overall accuracy is $96 \%$ obtained.

Thara and jasmine [14] discussed the brain tumor detection method based on Generalised Regression Neural Network and Probabilistic Neural Network. In this method Fuzzy c-means and K-means methods are used for image segmentation. They compared the performance of both methods for tumor detection and showed that Fuzzy c-means is better than K-means method. Direction and Magnitude are also obtained by adopting these methods.

\section{PROPOSED METHOD}

In this section implementation of the proposed work is presented in details with proposed algorithm and flowchart. BRATS 2013 dataset is used to evaluate the performance of the proposed method.

4.1 Proposed Algorithm

In the process of image segmentation various preprocessing steps have to be carried out so that brain tumor region can be extorted from MRI images. These steps are illustrated below.

Step-1: Select the 3-D MRI image from the BRATS 2013 data set.

Step-2: By using 3-D slicing, 3-D MRI image is converted into 2-D Format. The slices are required for extract the contents.

Step-3: Median Filter is used for noise reduction in MRI image.

Step-4: Contrast Adjustment of image gets saturated between low and high intensity values of the image.

Step-5: Image is divided into sub groups on the basis of intensity ranges.

Step-6: Canny edge detection approach is used for finding the sharp edges of the MRI image.

Step-7: Gray level co-occurrence matrix is obtained to extract the Texture features from MRI images.

Step-8: Feed Forward Neural Network (FFNN) is used for training process using Levenberg-Marquardt training function and accuracy is also measured with the help FFNN. 


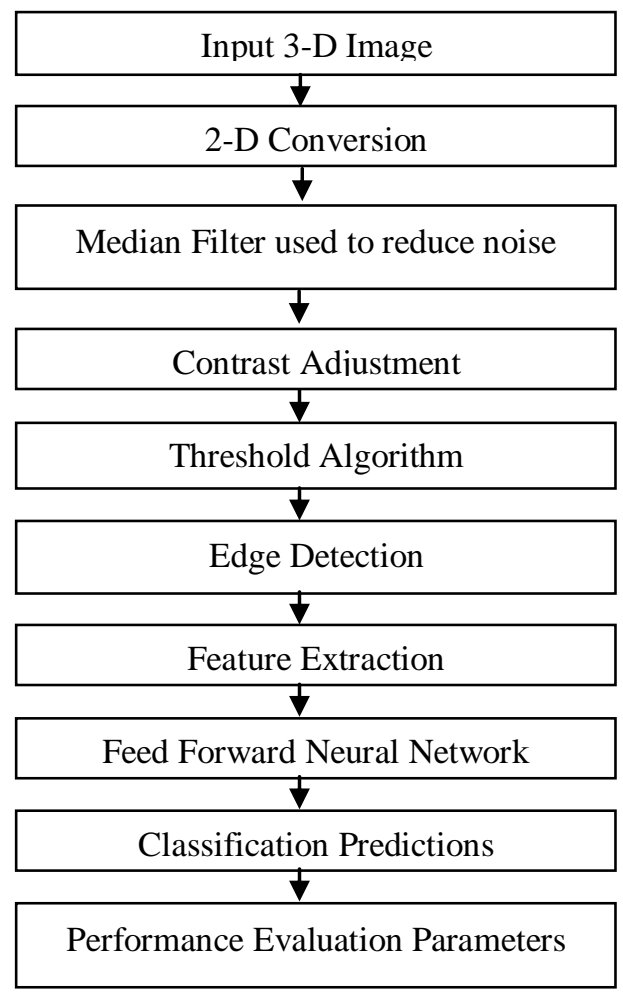

Figure 2: Flow Chart of the proposed method

\section{RESULT AND DISCUSSION}

In this section results of the proposed method is presented and discussed. In the proposed work brain tumor detection as well as classification has been done on the basis of feature values and segmentation process. Simulation of proposed work has been done in MATLAB 2013a. In this method image classification has been done using FFNN. Figure 3 shows the various MRI images used for tumor detection and classification process. BRATS 2013 dataset is used to evaluate the performance of the proposed method and dataset having 100 images with 75 MRI images are consist of tumor and 25 MRI images are not consist of tumor [15].

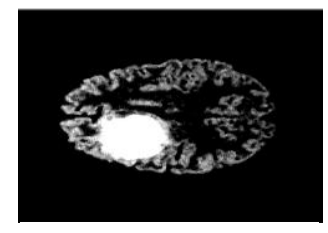

(a)

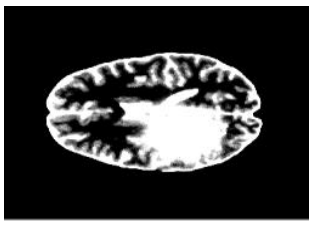

(e)



(i)

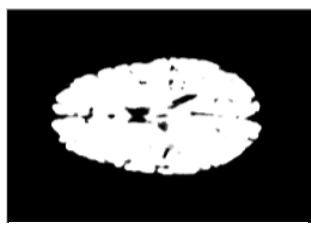

(b)

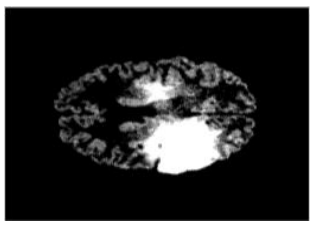

(f)

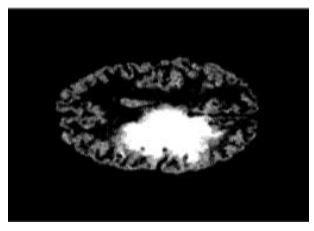

(i)

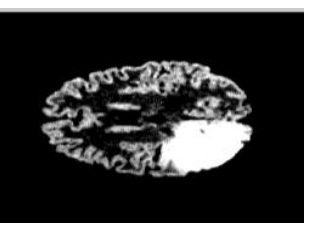

(c)

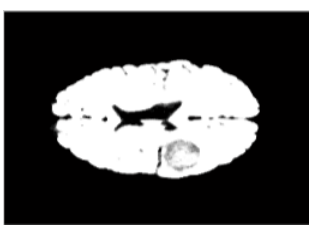

(g)

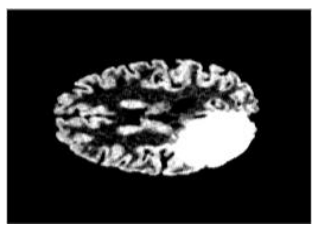

(k)

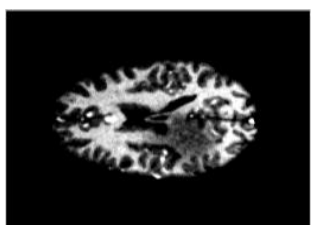

(d)

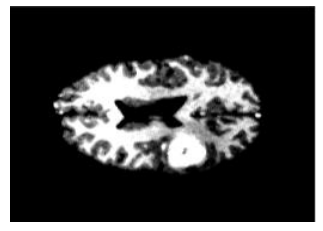

(h)

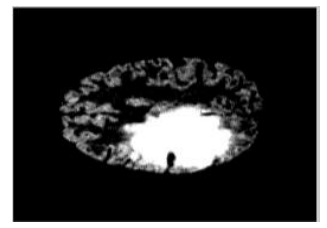

(1)

Figure 3: Original Images used for brain tumor detection in the proposed work from BRATS 2013 data set [15] 




(a)

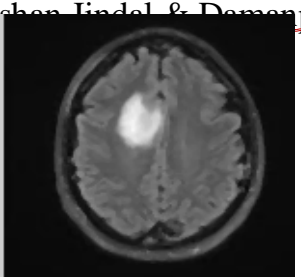

(b)

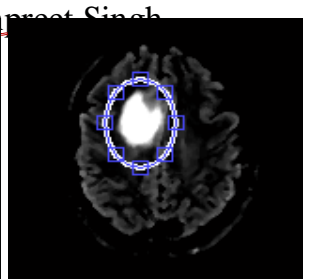

(c)

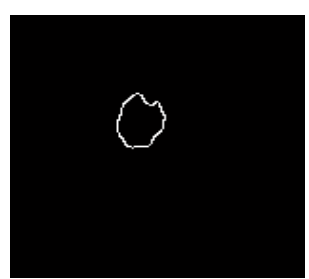

(d)

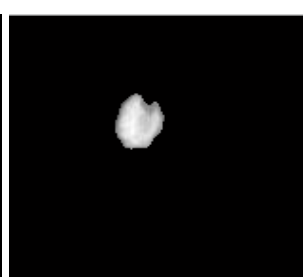

(e)

Figure 4: (a) MRI image of Brain (b) After noise reduction of MRI(c) Brain tumor detection in MRI (d) Edge detection in MRI (f) After segmentation of MRI

Figure 4 shows the brain tumor detection in magnetic resonance image. By using median filer noise is reduced in an image (b). The image (c) represents the detection of brain tumor. Segmentation and edge detection is also shown Figure 4.

\subsection{Performance Evaluation Parameters}

On the basis of classification of the tumor and non-tumor images various performance parameters Area, Perimeter, Contract, Homogeneity, Energy, Mean Square Error, Confusion Matrix and Overall Accuracy are measure to validate the proposed work.

\subsubsection{Feature Extraction}

Feature extraction is the process of minimizing the input data similar patterns etc. Texture is a useful feature for the medical image processing. In a gray level region it provides the spatial distribution for analysis. Various parameters are measured with the help of Gray level co-occurrence matrix.



Table 1 Experimental results of the proposed method for Brain Tumor detection in terms of Area, Perimeter, Contract, Homogeneity and Energy

\begin{tabular}{|l|l|l|l|l|l|}
\hline Image no. & \multicolumn{1}{|c|}{ Area } & \multicolumn{1}{|c|}{ Perimeter } & \multicolumn{1}{c|}{ Contract } & \multicolumn{1}{|c|}{ Homogeneity } & \multicolumn{1}{|c|}{ Energy } \\
\hline $\mathrm{a}$ & 246 & 420 & 0.4873 & 0.8905 & 0.4946 \\
\hline $\mathrm{b}$ & 150 & 131 & 0.1823 & 0.9646 & 0.5291 \\
\hline $\mathrm{c}$ & 345 & 365 & 0.5101 & 0.8914 & 0.4939 \\
\hline $\mathrm{d}$ & 180 & 170 & 0.2586 & 0.9107 & 0.4926 \\
\hline $\mathrm{e}$ & 485 & 423 & 0.4742 & 0.9035 & 0.4731 \\
\hline $\mathrm{f}$ & 319 & 292 & 0.4331 & 0.8949 & 0.4937 \\
\hline $\mathrm{g}$ & 138 & 122 & 0.1789 & 0.9629 & 0.5233 \\
\hline $\mathrm{h}$ & 153 & 145 & 0.2854 & 0.9069 & 0.4861 \\
\hline $\mathrm{i}$ & 229 & 201 & 0.3740 & 0.9120 & 0.4723 \\
\hline $\mathrm{j}$ & 398 & 692 & 0.4581 & 0.8909 & 0.4914 \\
\hline $\mathrm{k}$ & 363 & 609 & 0.4732 & 0.8958 & 0.4888 \\
\hline $\mathrm{l}$ & 448 & 432 & 0.4350 & 0.8986 & 0.4907 \\
\hline
\end{tabular}

In Table 1 shows various types of parameters, like Area, Perimeter, Contrast, Homogeneity and Energy detection with the help of gray level co-occurrence matrix of brain tumor and they gives effective results. Further these features are used for training of FFNN for classify the process. 
5.2 Mean Square Error (MSE)

Mean square error has been measure for computation of performance of proposed work. Error has been computed based on different classes that are predicted and that are actually available. With the help of MSE quality of the proposed work is also analyzed. Let $I$ is an image and $\mathrm{K}$ stands for noisy approximation.

$M S E=\frac{1}{m m} \sum_{i=0}^{m-1} \sum_{j=0}^{n=1}\left[I\left(i_{i}, j\right)-K\left(i_{i}, j\right)\right]^{2}$

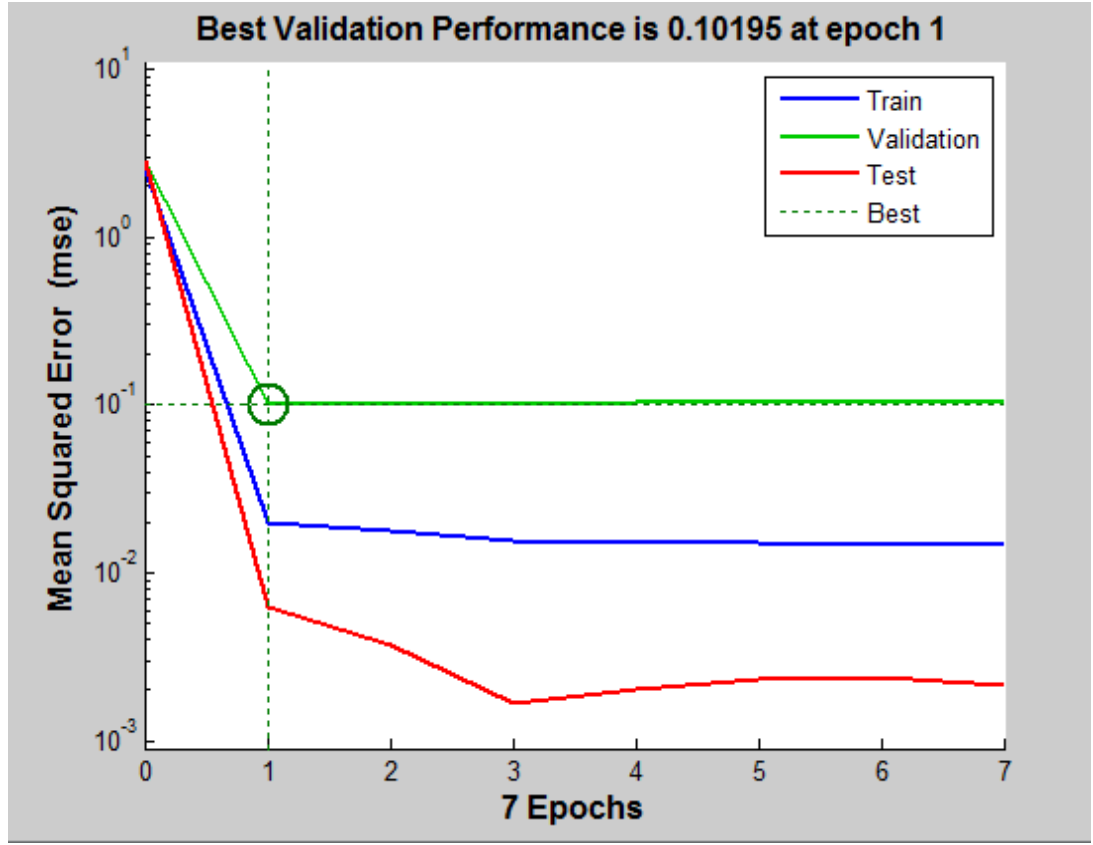

Figure 5: Mean Square Error for training, testing and validation process of the proposed method

Figure 5 shows error occurred during classification process using proposed method FFNN. On the basis of the training, validation and testing best performance has been computed 0.10195 at epoch 1 shown in Figure 5 and the value of MSE is always non-negative and value is closer to zero is better.

\subsection{Confusion Matrix}

Confusion Matrix is also known as Error Matrix that has been used for representation actual and predicted instances to different classes. False Positive, True Positive, False negative and True Negative has been used for representation confusion matrix. True positive are the term that are available in positive class and predicted in positive class. False positive represents the instances that are available in negative class but predicted in positive class.

$T P=\frac{\text { No. of images having brain tumor }}{\text { Total no, of images }}$

$T N=\frac{\text { No. of images withouttumor }}{\text { Total no, of images }}$

$F P=\frac{\text { No, of images falsely detected as tumor }}{\text { Total no, of images }}$

$F N=\frac{\text { No. of images not detected braîn tumor }}{\text { Total no. of images }}$

Accuracy $=\frac{(\mathrm{TP}+\mathrm{TN})}{(\mathrm{TP}+\mathrm{TN}+\mathrm{FN}+\mathrm{FP})}$ 


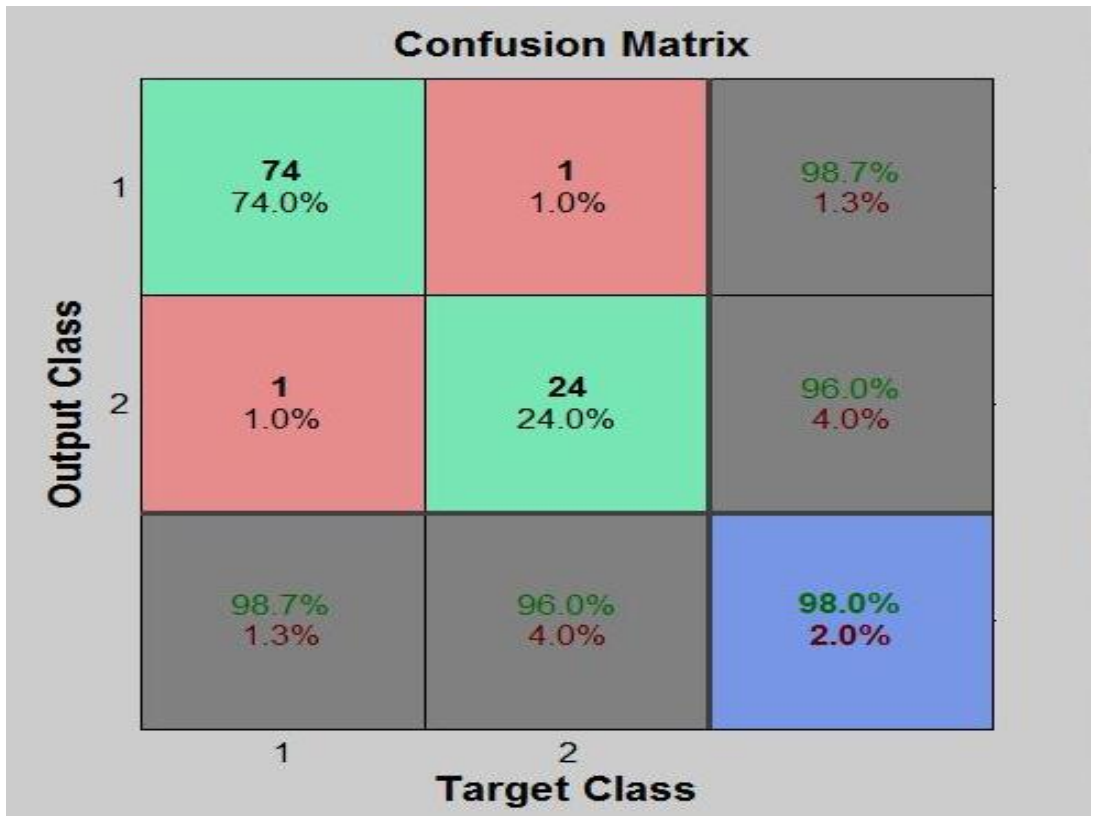

Figure 6: Confusion Matrix of the proposed method using Feed Forward Neural Network

Figure 6 shows Confusion Matrix based on image classification using FFNN. Confusion Matrix provides information about various instances and classes of the proposed method. There are four classes True Positive (TP), False Positive (FP), False Negative (FN) and True Negative (TN) in the Confusion Matrix. True Positive means number of resulted images having brain tumor, False Positive means number of images falsely detected as tumor, False Negative means number of images having brain tumor and not detected and True Negative means number of images without tumor is shown in Figure 6. Accuracy of the proposed work can be analyzed with the help of these classes. It has been concluded that in Confusion Matrix out of 100 instances 98 instances are correctly classified and 2 instances that are incorrectly classified. On the basis of Confusion Matrix it is concluded that the accuracy of the proposed work is $98 \%$.

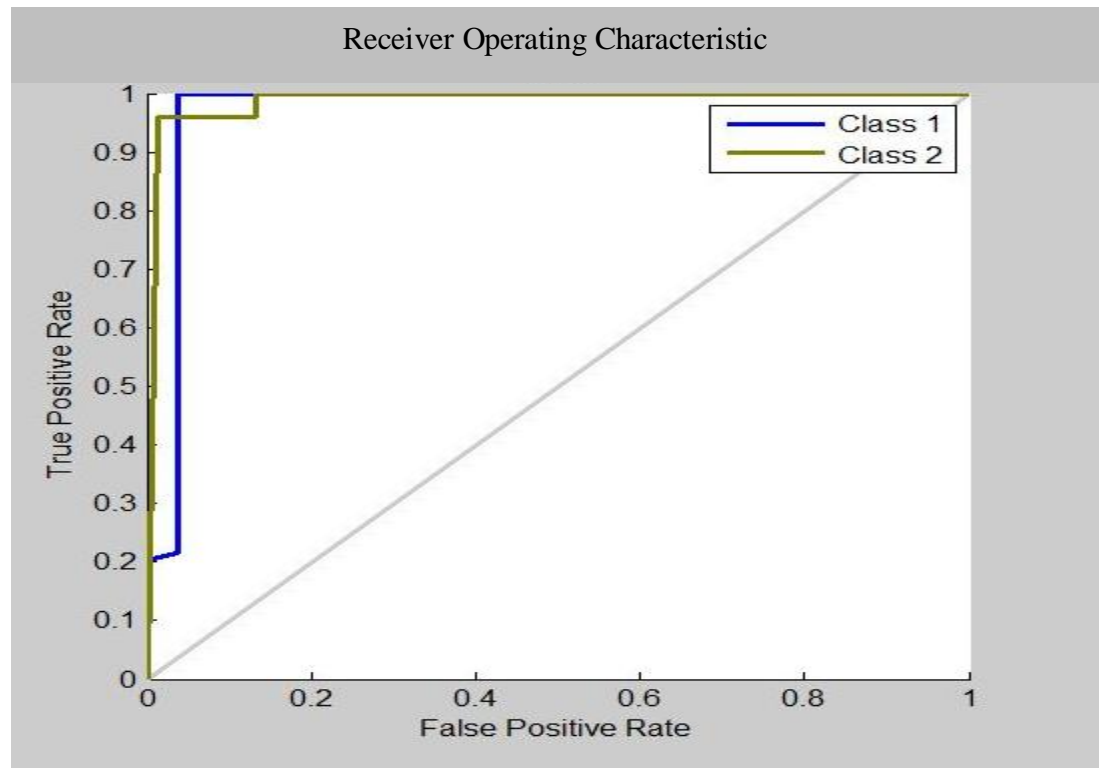

Figure 7: Show the graph between class-1 and class-2 of Receiver Operating Characteristic

Figure 7 shows Receiver Operating Characteristic (ROC) analysis used in demonstrating the binary classifier performance by varying its discrimination using False Positive Rate (FPR) and True Positive Rate (TPR). The False Positive Rate is called fall 
out or probability of false alarm and True Positive Rate is called sensitivity or probability of detection. Class 1 show True Positive Rate and class 2 show False Positive Rate.

Table 2 show the comparison between Back Propagation Neural Network method (BPNN), Radial Basis Function (RBF) method, Adaptive Boosting Classifier (ADABOOST) method, Support Vector Machine (SVM) and Proposed Method for tumor detection in the brain. From Table 2, it has been concluded that the proposed approach provide better classification as compare to existing approaches.

Table 2 Comparison of the proposed method with Back Propagation Neural Network, Radial Basis Function, Adaptive Boosting Classifier and Support Vector Machine for Brain Tumor Detection

\begin{tabular}{|l|l|}
\hline Approaches & Accuracy in $\%$ \\
\hline BPNN & 75 \\
\hline RBF & 85.71 \\
\hline ADABOOST & 90.11 \\
\hline SVM & 95.6 \\
\hline Proposed Method & 98.0 \\
\hline
\end{tabular}

\section{CONCLUSION \& FUTURE SCOPE}

In this work, MRI images are used for classification of dataset that contain tumor and non-tumor in MRI images. Gray level co-occurrence matrix is required for computation of energy, contrast and homogeneity. Further these features are used for training the Feed Forward Neural Network and classify the process. The proposed work helps in detecting the tumor using Feed Forward Neural Network but in future it can be used for real world application for brain tumor detection from the patients. To develop effective Neural Network classification, Machine leveling algorithm can be used for optimizing the input weights for hidden layers to increase the brain tumor detection accuracy. From the experimental results it has been concluded that the performance of the proposed method is better than existing approaches in terms of accuracy.

\section{REFERENCES}

[1] P. Natarajan, N. Krishnan, S. Kenkre, S. Nancy and B. Singh, "Tumor Detection using threshold operation in MRI Brain Images", Proc. of the IEEE International Conference on Computational Intelligence and Computing Research, 18-20 Dec. Coimbatore, India. pp. 1-4, 2012.

[2] D. Somwanshi, A. Kumar, P. Sharma, and D. Joshi, "An efficient Brain Tumor Detection from MRI Images using Entropy Measures", Proc. of the IEEE International Conference on Recent Advances and Innovations in Engineering (ICRAIE), 23-25 Dec. Jaipur, India. pp. 1-5, 2016.

[3] A. Nandi, "Detection of human brain tumor using MRI image segmentation and morphological operators", Proc. of the IEEE International Conference on Computer Graphics, Vision and Information Security (CGVIS), 2-3 Nov. Bhubaneswar, India. pp. 55-60, 2015.

[4] https://www.brain-surgery.com accessed on April, 2017.

[5] P. G.B, and A. Agrawal, "Hybrid Approach for Brain Tumor Detection and Classification in Magnetic Resonance Images", Proc. of the International Conference on Communication, Control and Intelligent Systems, 7-8 Nov. Mathura, India. pp. 162-166, 2015.

[6] http://www.abta.org accessed on April-May, 2017.

[7] http://www.braintumor.org accessed on April-May, 2017.

[8] H. Kaur, and R. Bhatia, "Medical Image Segmentation using Penalized FCM and Pollination Based Optimization Approach", International Journal of Advanced Research in Computer Science and Software Engineering 118(4), 8 Aug. pp. 602-608, 2015.

[9] A. Kaur, "An Automatic Brain Tumor Extraction Systems using Different Segmentation Methods", Proc. of the Second International Conference on Computational Intelligence \& Communication Technology, 12-13 Feb. Ghaziabad, India. pp. 187-191, 2016.

[10] A. Sehgal, S. Goel, P. Mangipudi, A. Mehra and D. Tyagi, "Automatic Brain Tumor Segmentation and Extraction in MR Images", Proc. of the Conference on Advances in Signal Processing Cummins College of Engineering for Women, 9-11 Jun. Pune, India. pp. 104-107, 2016

[11] K. Bhima and A. Jagan, "Analysis of MRI based Brain Tumor Identification using Segmentation Techniques", Proc. of the International Conference on Communication and Signal Processing, 6-8 Apr. Tamil Nadu, India. pp. 2109-2113, 2016.

[12] C. Li, Y. Li, Li and X. Wu, "Novel Fuzzy C-Means Segmentation Algorithm for Image with the Spatial Neighborhoods", Proc. of the IEEE $2^{\text {nd }}$ International Conference on Remote Sensing, Environment and Transportation Engineering (RSETE). 1-3 Jun. Nanjing, China. pp. 1- 4, 2012.

[13] N. Al-Azzawi and M. Sabir, "An Superior Achievement of Brain Tumor Detection Using Segmentation Based on F-Transform", Proc. of the IEEE International Conference on Computer Networks and Information Security, 19-21 Sept. Tunisia, North Africa. pp. 1-6, 2015.

[14] K.S. Thara and K. Jasmine, "Brain Tumor Detection in MRI Images using PNN and GRNN", Proc. of the IEEE Wireless Communications, Signal Processing and Networking, 23-25 Mar. Chennai, India. pp. 1504-1510, 2016.

[15] MICCAI Brats 2013 http://martinos.org/qtim/miccai2013/data.html/ downloaded on 10 Oct, 2016. 